\title{
A reflection on mathematical modeling in tissue engineering
}

\author{
Abstract \\ This paper highlights some of the issues connected to mathematical modeling with \\ applications to tissue engineering where injection of stem cells is of importance.
}

Volume I Issue 2 - 2016

\author{
Vermolen FJ \\ Delft Institute of Applied Mathematics, Delft University of \\ Technology, Netherlands
}

\begin{abstract}
Correspondence: Vermolen FJ, Delft Institute of Applied Mathematics, Delft University of Technology, Netherlands, Email f.j.vermolen@gmail.com
\end{abstract}

Received: December 0I, 2016 | Published: December 14, 2016

\section{Opinion}

Mathematical modeling in the field of biology becomes more and more powerful with the increasing growth of computational capacity. Nowadays processes like wound healing, development of ulcers, organs and cancer are simulated to aid medical biologists and tissue engineers. This form of mathematical modeling is often received with either skepticism or with an oblivious amount of confidence of computed results. Therefore it is good to reflect on mathematical models as such and on computed results.

If we go back to history of modern exact sciences, then we come across people like Euler, Newton, Pascal, Huygens, Stevin, Dirac and later on people like Einstein, Schrödinger and Heissenberg. Most of the scientists in the earlier ages were combining sciences like mathematics and physics. To this extent, the collaboration between physics and mathematics has existed for many centuries. One of the many important contributions in physics was the formulation of Newton's Law, which according to the tales, followed from the observation that an apple falls down as it gets de-attached from the tree. Newton never observed an apple that hovered through the sky or that would fly through the sky (unless a big bird would have taken it). He further observed that inertia is important, and to this end, he came up with his important Law

$$
F=m a,
$$

where $\mathrm{F}, \mathrm{m}$ and $\mathrm{a}$, respectively, represent the force, mass and acceleration of an object. Here F can also be interpreted as a (vectorial) sum over all forces. Later Einstein introduced relativity and based on observations, he postulated that the mass and dimensions of a body are influenced by its speed with reference to another coordinate system. In particular if speeds approach the speed of light, then this effect becomes quite significant. Hence what we see is that the accepted formalisms evolve over time.

Mathematical modeling in tissue engineering is rather new as it all started several decades ago. The first models were based on insights that existed before and currently new insights are developed resulting from present day discoveries. Of course this is a good thing since modeling contributes to understanding the process. Understanding such a process develops by carrying out experiments, both in vitro (on laboratory samples) and in vivo (on actual patients in a clinical setting). Understanding involves the formulation of hypotheses and in order to validate hypotheses against experimental results, these hypotheses need to be quantified. Hence mathematical relations are developed and therefore in this way, mathematical models are born. These models will contain errors in terms of the formulation of wrong hypotheses by seeing the wrong patterns in the observation space. Further, the quantification, or development of mathematical theory to describe the experiments or process could have been carried out wrongfully. Therefore, it is of crucial importance to evaluate the mathematical models, which have been proposed. This evaluation should be done in terms of existence (does the problem have a solution at all?), uniqueness (is there only one solution, or just one description of the process?) and stability (does a small variation also have a small impact?) of a solution to such a mathematical problem. If these criteria are satisfied, then mathematicians call the problem well-posed. If not, then, the modeler has to revise the formulation. Suppose that the model is well-posed, then numerical scheme often has to be developed to actually calculate the solution. Furthermore, the right parameter values have to be introduced into the model. This last issue poses a major challenge, as well as a large and dangerous threat to modeling at all since it is very hard to get the right parameter values. Most of the input parameters are not directly measurable and to this extent, parameter fitting through regression methods is indispensable. It is also important to quantify the impact of the input parameters on the output simulations. Do variations of input values lead to completely different behavior in the simulations? In other words, does the qualitative behavior change (completely)? Or do these variations only lead to qualitative changes in the sense that the simulation patterns stay more or less the same?

Another issue concerns the development of the numerical scheme to approximate the solution of the mathematical problem. Questions on the reliability are crucially important. Here, one can ask the same questions whether the obtained approximation, also referred to as the numerical solution, reflects the behavior that is to be expected from the mathematical analysis as well as from the experimental observations. Numerical stability and accuracy are very important principles and modelers should be aware of the limitations and reliability of the computational methods and simulation results. Numerical errors come in due to truncation and rounding of (real-valued) numbers. 
The construction of realistic models, that is, models that can be used to reproduce the most important characteristics, often requires a trade-off between complexity and simplicity. Complex models are able to incorporate many features, and as such this class of models stays very close to biology and physics. However, these models often require the quantification of many input parameters, which are hard to obtain, and hence these models introduce a large degree of uncertainty. The more simple models often require fewer input parameters and therewith they have the advantage of being tractable and therewith it is often easy to determine unknown parameters from regression procedures using experimental observations. Then these models can be used to predict certain phenomena like the occurrence of a serious hypertrophic scar after a burn injury or the spread of cancer given certain circumstances. A drawback of this class of models is the low amount of physics involved and thereby this class of models may have limited applicability if it comes to understanding a process. However, its predictability is much better and easier. The complicated models involve more physical aspects and there with these models are very useful for the actual understanding a certain process.

\section{Acknowledgements}

None.

\section{Conflict of interest}

The author declares no conflict of interest. 